\title{
Monitoring Media Content About Vaccines in the United States: Data from the Vaccine Sentimeter
}

\author{
Guido A. Powell ${ }^{\star 1}$, Kate Zinszer ${ }^{1}$, Aman D. Verma', Lawrence C. Madoff ${ }^{3}$, Chi Bahk², \\ John S. Brownstein ${ }^{4}$ and David Buckeridge ${ }^{1}$
}

${ }^{1}$ Epidemiology, McGill University, Montreal, QC, Canada; ${ }^{2}$ Epidemico, Boston, MA, USA; ${ }^{3}$ University of Massachusetts Medical School, Worchester, MA, USA; ${ }^{4}$ Computational Epidemiology Group, Children's Hospital Informatics Program, Devision of Emergency Medicine, Boston Children's Hospital, Boston, MA, USA

\section{Objective}

The current analysis describes the scope and trends in United States content from the Vaccine Sentimeter's results, while seeking to examine any possible links between media content, vaccine coverage, and reported vaccine adverse events in the country.

\section{Introduction}

The success of public health campaigns in decreasing or eliminating the burden of vaccine-preventable diseases can be undermined by media content influencing vaccine hesitancy in the population. A tool for tracking and describing the ever-growing platforms for such media content can help decide how and where to invest in campaigns to increase public confidence in vaccines. The Vaccine Sentimeter, developed from the Healthmap project, aims to assist public health practitioners in maintaining or improving vaccine coverage through a real-time, online visualization tool of global media content on vaccines.

\section{Methods}

The Vaccine Sentimeter collected over 9,552 online articles covering US events from 2012 to 2014 , from sources such as news aggregators, government reports, and blogs. The sources were searched every hour using key terms related to vaccines, such as brand names, adverse events, or vernaculars (e.g. "shots"). Through both manual and automated curation, articles were classified by date, location, vaccine, category (topic such as "outbreaks","research", "costs", etc.), and overall article sentiment (positive, neutral/unclear, or negative). Data on vaccine coverage rates were obtained online from the results of the Center for Disease Control's National Immunization Survey (NIS), the Behavioral Risk Factor Surveillance System (BRFSS), and the National Health Interview Survey (NHIS). Reports of adverse events were collected from online data of the Vaccine Adverse Event Reporting System (VAERS). National coverage for 12 vaccines for which sufficient reporting was found in the Vaccine Sentimeter articles were examined for possible links to the sentiment of media content on each vaccine. Finally, the categories of media content were compared to that of the VAERS to examine whether the media content about adverse events and other topics followed similar patterns as those reported in the public.

\section{Results}

The three most frequently reported states in the media were California, New York, and Texas with, respectively, 760, 517, and 393 classified reports. Most frequently reported vaccines included Influenza/H1N1 vaccines ( $\mathrm{n}=2750)$, human papillomavirus vaccines (HPV) ( $\mathrm{n}=1678)$, vaccines of the mumps, measles, and/or rubella family (MMR) $(n=1363)$, vaccines of the diphtheria, pertussis, and/or tetanus (DPaT) family $(\mathrm{n}=847)$, and polio-related vaccines $(\mathrm{n}=450)$. Across the US, $10.2 \%$ of vaccine media content was classified as being of an overall negative sentiment, while $86.4 \%$ were positive and 3.4\% neutral/unclear. Most frequent classified categories included policy recommendations $(\mathrm{n}=1365)$, delivery strategies $(n=907)$, outbreaks $(n=756)$, and vaccine effectiveness $(n=617)$. National coverage for vaccines of interest ranged from $57 \%$ (HPV) to $92.9 \%$ (polio vaccines). The percentage of negative sentiment for these vaccines ranged from $1.6 \%$ (meningococcal vaccines) to $21.5 \%$ (Hepatitis B vaccines). National vaccine coverage was negatively but not significantly correlated $(r=-0.47, p=.122)$ with percentage of negative media sentiment for the 12 vaccines of interest.

\section{Conclusions}

These and future findings emphasize the importance of the Vaccine Sentimeter as a valid tool for public health agencies. This tool may allow them to track any loss of confidence in a variety of vaccines, at either the national or state level, at an early enough stage to allow effective policy implementation.

Funding for development of the Vaccine Sentimeter was provided by Sanofi-Pasteur

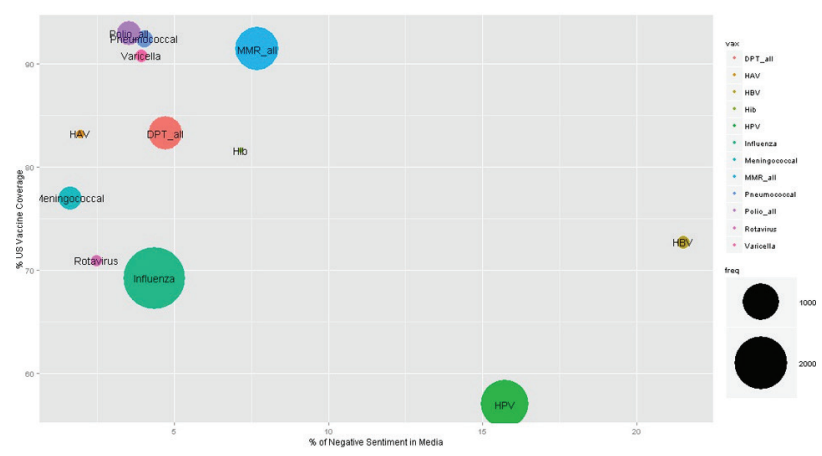

Vaccination coverage for 12 vaccines in the US plotted over the percentage of negative sentiment in media content

\section{Keywords}

Media content; Vaccine; HealthMap; Vaccine coverage; Vaccine Sentimeter

\section{*Guido A. Powell}

E-mail: gdpwll@gmail.com 